\title{
ORIGINAL
}

\section{CONSUMO ALIMENTAR DE ADOLESCENTES MATRICULADOS EM UM COLÉGIO PARTICULAR DE TERESINA, PIAUÍ, BRASIL}

\author{
FOOD CONSUMPTION BY ADOLESCENTS ENROLLED IN \\ A PRIVATE HIGH SCHOOL IN THE CITY OF TERESINA, PIAUÍ, BRAZIL
}

\author{
Cecilia Maria Resende Gonçalves de CARVALHO' \\ Apolonia Maria Tavares NOGUEIRA' \\ João Batista Mendes TELES ${ }^{2}$ \\ Suzana Maria Rebêlo da PAZ ${ }^{3}$ \\ Rosângela Maria Lopes de SOUSA ${ }^{3}$
}

\begin{abstract}
RESUMO
Com o objetivo de estudar o consumo alimentar de adolescentes matriculados em um colégio particular de Teresina, realizou-se estudo transversal através de amostragem sistemática. A freqüência de consumo de alimentos expressa em dias por semana foi obtida mediante questionário administrado individualmente ao aluno. O estado nutricional foi avaliado pelo Índice de Massa Corporal, considerando com sobrepeso valores $\geq P 85$. Os resultados indicam prevalência elevada de risco para o sobrepeso $(19,8 \%)$ nos 334 adolescentes. Os meninos consumiram com maior freqüência alimentos energéticos (milho, manteiga, pizza, lasanha, sucos industrializados, refrigerantes) e construtores (frango, ovos, leguminosas) em relação às meninas ( $p<0,05)$. Os adolescentes de 10 a 13 anos consumiram com maior freqüência milho, mel, açúcar, rapadura enquanto aqueles com idade entre 17 e 19 anos consumiram mais arroz, bolos, biscoitos, rosca, balas, goma de mascar, refrigerantes, frutas verde-amarelo.
\end{abstract}

Unitermos: consumo de alimentos, adolescente, índice de massa corporal, escola particular.

\begin{abstract}
Food consumption by adolescents enrolled in a private high school in the city of Teresina, Piauí, was evaluated in a systematic sampling cross-sectional study. The food consumption frequency in days per week was obtained using an individually filled out questionnaire. The nutritional status was evaluated through the body mass index, considering as overweight data $\geq P 85$. The results show a high overweight risk prevalence (19.8\%) in the 334 adolescents. The boys consumed with higher frequency energetic food (corn, butter, pizza, lasagna, industrialized juices, soft drinks) and constructors (chicken, eggs, vegetables) in relation to the girls $(p<0.05)$. The adolescents between ten and thirteen years old consumed with higher frequency corn, honey, sugar, raw brown sugar, while the ones with age between seventeen and nineteen years old consumed more rice, cakes, cookies, rusk, candies, chewing gum, soft drinks, green-yelow fruits.
\end{abstract}

Index terms: food consumption, adolescent, body massa index, private high school.

\footnotetext{
(1) Departamento de Nutrição, Universidade Federal do Piauí, Centro de Ciências da Saúde. Campus Iningá, SG 13, 64050-500, Teresina, PI, Brasil. Correspondência para/Correspondence to: C.M.R.G. CARVALHO.E-mail: cecilia@webone.com.br

(2) Departamento de Medicina Comunitária, Universidade Federal do Piauí.

(3) Curso de Graduação em Nutrição, Universidade Federal do Piauí.
} 


\section{INTRODUÇÃO}

Adolescência é o período da vida que vai dos 10 aos 19 anos 11 meses e 29 dias, segundo critérios cronológicos propostos pela Organização Mundial de Saúde (World Health..., 1995), e se caracteriza por profundas transformações somáticas, psicológicas e sociais.

Durante a adolescência, especialmente na puberdade, ocorre acentuado crescimento físico, período em que aumenta $50 \%$ do peso e $15 \%$ da estatura final do adulto. O crescimento acelerado, acompanhado pelo desenvolvimento psicossocial e estimulação cognitiva intensa, torna as necessidades de energia e nutrientes elevadas, e na maioria das vezes, são inadequadamente atendidas.

As mudanças observadas no hábito alimentar da população brasileira, em função de fatores diversos, têm favorecido o aparecimento da obesidade, doenças do coração, câncer e outras enfermidades (Monteiro \& Mondini, 1995; World Health..., 1997), que vêm se constituindo em importantes causas de morte em muitos países e também no Brasil.

A obesidade na infância e na adolescência tem como conseqüência a possibilidade de sua manutenção na vida adulta, levando a uma situação de risco à saúde e ao aumento da morbi-mortalidade (Must et al., 1992; Guo et al., 1994). Dados recentes apontam a obesidade como um dos problemas nutricionais mais prevalentes nos Estados Unidos, chegando a afetar um terço dos adolescentes. Na Espanha, cerca de 10 a 20\% dos adolescentes são obesos (Anta et al., 1996), e no Brasil, já é prática comum na alimentação desses jovens o consumo de lanches e fast foods, geralmente associado a dietas nutricionalmente inadequadas, apresentando alta quantidade de energia, com carência de ferro, cálcio, vitaminas A e C.

$\mathrm{O}$ aumento da obesidade e suas seqüelas como problema clínico e social tem estimulado a realização de estudos com adultos, no sentido de se conhecer o problema e poder contribuir para sua prevenção e tratamento. Sem embargo, poucos estudos têm sido conduzidos com adolescentes, cujo hábito alimentar tende a sofrer relativas mudanças, entre as quais se observa o aumento no consumo de lanches gordurosos, ricos em açúcares e proteínas, pobres em cálcio, ferro e fibras pelo baixo consumo de frutas, vegetais, peixes, leite e cereais integrais (Mcpherson et al., 1990; Huang et al., 1994; Pérez-Liamas, et al., 1996; Watt \& Sheiham, 1996).

Estudos no Brasil relacionados ao consumo alimentar de adolescentes, indicam baixa ingestão de produtos lácteos, frutas, hortaliças, alimentos fontes de proteína e ferro e excesso de açúcar e gordura (Lerner, 1994; Gambardella, 1996; Gambardella et al., 1999).

O reconhecimento precoce de práticas alimentares incorretas pode estimular o interesse dos dirigentes, estudantes e familiares pela implantação de programas de educação alimentar, com vistas à adoção de medidas corretivas dirigidas especialmente aos escolares, para obtenção de uma dieta adequada às suas reais necessidades, e que favoreça a prevenção de determinadas doenças.

Devido à falta de estudos em escolares adolescentes piauienses, decidiu-se realizar esta investigação, objetivando conhecer o perfil do consumo alimentar em uma amostra constituída pelos adolescentes matriculados em um colégio particular de Teresina.

\section{MATERIAL E MÉTODOS}

O universo do estudo foi constituído pelos adolescentes de 10 a 19 anos, matriculados na rede privada de ensino em Teresina. Para representar esse universo foi pesquisada uma escola que voluntariamente aceitou participar da investigação. Do total de 862 adolescentes matriculados nessa escola no ano de 1996, foram selecionados 360 , por amostragem sistemática, com intervalos de sistematização igual a 3, ou seja, de cada três adolescentes, um foi incluído na pesquisa, sendo que o primeiro foi escolhido aleatoriamente entre os três primeiros números da lista fornecida pela direção da escola. Esse tamanho amostral embute um erro de $5 \%$ e um nível de significância de 95\%, nos estimadores de proporção (\%).

Efetivamente foram coletados dados de 334 adolescentes, de ambos os sexos, que representam uma fração amostral de 5,89\% do universo de 5662 adolescentes matriculados em cinco colégios da rede privada de ensino, cujos alunos pertencem aos mesmos estratos sociais. Os dados foram coletados no momento de realização da prática de educação física e, de acordo com os dados registrados na Ficha de Cadastro do Aluno, não existia referência a doenças entre os adolescentes incluídos no estudo. A exclusão de 26 estudantes ocorreu em virtude dos mesmos terem sido transferidos para outros colégios ou por estarem ausentes no dia da realização da coleta dos dados. Após três tentativas foram considerados não resposta (7,22\% da amostra).

Os adolescentes selecionados aceitaram participar voluntariamente e tiveram autorização prévia da direção da escola, antes de serem incluídos na pesquisa. O protocolo deste estudo foi aprovado pelo Comitê de Ética da Universidade Federal do Piauí.

\section{Consumo alimentar}

O consumo alimentar dos adolescentes foi investigado utilizando-se o questionário de freqüência do consumo de alimentos tendo em vista seu baixo custo e praticidade de aplicação. Os questionários contendo a listagem dos alimentos foram estruturados em três partes, de acordo com a classificação dos alimentos em três grupos distintos: Alimentos construtores (leite e produtos lácteos, carnes, ovos, miúdos/vísceras e leguminosas); Alimentos reguladores (hortaliças e frutas) e Alimentos energéticos (cereais, raízes, tubérculos, pães, massas, bolos, gorduras, açúcares, doces e bebidas açucaradas). Em cada 
um dos grupos de alimentos determinou-se o consumo alimentar de duas maneiras: uma classificando-se em freqüência semanal, e outra o consumo de cada alimento foi quantificado recebendo os valores a seguir: zero, quando o indivíduo informou que não consumia; um, para consumo menor que uma vez por semana; dois, para consumo de uma a duas vezes por semana; três, para consumo de 3 a 4 vezes por semana e, quatro, para consumo de cinco ou mais vezes por semana. Os questionários foram testados e padronizados antes de serem preenchidos, individualmente, pelos alunos, que foram devidamente orientados sobre a maneira correta de preenchimento.

\section{Antropometria}

Para melhor caracterizar a população-alvo desta investigação tomaram-se as medidas de peso e altura, as quais foram realizadas com os indivíduos descalços, em balança tipo plataforma, com capacidade para até $160 \mathrm{~kg}$ e sensibilidade de 100 g. A altura foi obtida com os estudantes em posição ereta, descalços, pés unidos e em paralelo, utilizando-se a trena metálica com esquadro que se encontra acoplada à balança. O esquadro foi firmemente apoiado sobre a cabeça, subseqüentemente, assegurando-se que o estudante encontrava-se na posição correta para proceder-se à leitura e registro da altura obtida.

Para avaliação do estado nutricional, adotou-se o critério da World Health... (1995), que estabelece o equacionamento da condição nutricional de acordo com o percentil ocupado pelo valor do Índice de Massa Corporal para uma determinada idade (IMC/Idade); segundo o referido critério, IMC/Idade $<$ P5 é indicativo de magreza; IMC/Idade S P5 e < P85 corresponde à eutrofia; e IMCIdade $\geq$ P85 representa risco para sobrepeso.

\section{Análise dos dados}

Os dados coletados foram tabulados e analisados pelo software for Windows SPSS 8.0 e pelo software
StatXact-3. Os resultados foram apresentados em tabelas que expressam a freqüência média do consumo semanal de alimentos distribuídos em três grupos (energéticos, reguladores e construtores).

As médias das freqüências e o desvio-padrão do consumo semanal de alimentos, por sexo e faixa etária, foram comparadas aplicando-se a técnica de análise de variância (Anova), considerando-se o nível de significância de $95 \%$.

Nos testes de associação quando à freqüência observada foi menor do que cinco; as categorias da variável foram reagrupadas e o teste foi feito com o Qui-quadrado de Pearson constante no software StatXact-3.

A associação entre o IMC e o sexo dos adolescentes foi avaliada utilizando-se o Qui-quadrado (razão de verossimilhança), considerado significativo quando o valor do $p$ ( $p$-value) for menor que 0,05.

\section{RESULTADOS}

Dos 360 estudantes selecionados, 334 participaram do estudo. A amostra foi composta de 153 meninos (45,8\%) e 181 meninas (54,2\%), cuja idade média variou entre $13,2 \pm 2,0$ e $13,8 \pm 2,2$ anos, respectivamente. A estatura média dos meninos foi 1,56 $\pm 0,12 \mathrm{~m}$ e das meninas $1,54 \pm 0,08 \mathrm{~m}$. O peso médio para os meninos foi de $49,9 \pm 13,0 \mathrm{~kg}$ e para as meninas $49,1 \pm 11,7 \mathrm{~kg}$. Para o total da amostra o IMC mínimo foi 15,1 e o máximo 32,11.

Apesar da maioria dos adolescentes apresentar eutropia quanto ao índice de massa corporal, o risco para o sobrepeso atingiu cerca de 20\% nos 334 estudantes investigados. Em contrapartida, o baixo peso não alcançou o limite esperado que é de $5 \%$. O teste do Qui-quadrado não revelou associação entre o estado nutricional e o sexo dos adolescentes ( $p>0,05)$. Os maiores percentuais de sobrepeso (IMC $\geq$ P85) foram encontrados na população masculina (24,8\%), situação encontrada em apenas 15,5\% das meninas (Tabela 1).

Tabela 1. Sobrepeso em estudantes adolescentes de acordo com o sexo.

\begin{tabular}{|c|c|c|c|c|c|c|}
\hline \multirow{2}{*}{ Estado Nutricional } & \multicolumn{2}{|c|}{ Masculino } & \multicolumn{2}{|c|}{ Feminino } & \multicolumn{2}{|c|}{ Total } \\
\hline & $\mathrm{n}$ & $\%$ & $\mathrm{n}$ & $\%$ & $\mathrm{n}$ & $\%$ \\
\hline $\begin{array}{l}\text { Normalidade } \\
(\mathrm{P} 5 \leq \mathrm{IMC}<\mathrm{P} 85)\end{array}$ & 110 & 71,9 & 148 & 81,7 & 258 & 77,2 \\
\hline $\begin{array}{l}\text { Risco para sobrepeso } \\
(I M C P \geq P 85)\end{array}$ & 38 & 24,8 & 28 & 15,5 & 66 & 19,8 \\
\hline $\begin{array}{l}\text { Magreza } \\
(\mathrm{P} 5<\mathrm{IMC})\end{array}$ & 5 & 3,3 & 5 & 2,8 & 10 & 2,3 \\
\hline Total & 153 & 45,8 & 181 & 54,2 & 334 & 100,0 \\
\hline
\end{tabular}

$\mathrm{p}=0,0995$ (Teste Qui-quadrado). 
A partir dos dados da Tabela 2, pode-se perceber que, dos alimentos-fonte de proteínas (grupo dos construtores), o feijão ocupa posição de destaque. Entre 80 e $90 \%$ dos pesquisados informaram consumir o alimento pelo menos uma vez por semana. As outras leguminosas que apareceram foram a lentilha e a ervilha, com freqüência bem menor, especialmente entre as meninas $(p<0,05)$ (Tabela 3).

Quanto às carnes, merece destaque a carne bovina, seguida do frango para os meninos e de leite/queijo para

Tabela 2. Freqüência do consumo semanal (ao menos uma vez na semana) dos grupos de alimentos pelos adolescentes de ambos os sexos.

\begin{tabular}{|c|c|c|}
\hline Grupo Alimentar & Masculino & Feminino \\
\hline \multicolumn{3}{|l|}{ - Construtores } \\
\hline Carne bovina & 65,99 & 67,95 \\
\hline Frango & 62,26 & 50,01 \\
\hline Lingüiça & 40,39 & 33,71 \\
\hline Peixe & 26,36 & 23,95 \\
\hline Leite/queijo & 51,86 & 54,55 \\
\hline logurte & 37,16 & 36,36 \\
\hline Ovos & 56,64 & 23,63 \\
\hline Leguminosas (feijão) & 90,16 & 84,09 \\
\hline Outras leguminosas & 29,55 & 16,56 \\
\hline \multicolumn{3}{|l|}{ - Energéticos } \\
\hline Arroz & 91,94 & 92,74 \\
\hline Macarrão & 70,35 & 73,45 \\
\hline Cuscuz de milho & 64,48 & 67,76 \\
\hline Batata/Inhame & 43,03 & 38,53 \\
\hline Aveia (farinha) & 42,76 & 33,52 \\
\hline Beiju (tapioca) & 55,15 & 59,26 \\
\hline Pão branco & 88,59 & 87,77 \\
\hline Bolo/biscoito/rosca & 77,03 & 88,40 \\
\hline Pastel/coxinha/bomba & 72,16 & 71,67 \\
\hline Margarina/manteiga & 64,59 & 58,98 \\
\hline Pizza/lasanha & 56,00 & 47,17 \\
\hline Mel & 49,34 & 37,36 \\
\hline Açúcar comum & 87,43 & 50,39 \\
\hline Chocolate (tablete) & 64,19 & 63,43 \\
\hline Bombom & 80,67 & 88,95 \\
\hline Rapadura & 29,33 & 18,46 \\
\hline Balas/goma de mascar & 79,34 & 86,04 \\
\hline Gelatinas/pudins/cremes & 56,53 & 57,95 \\
\hline Sorvetes & 68,92 & 77,54 \\
\hline Sucos industrializados & 48,63 & 32,24 \\
\hline Refrigerantes & 94,70 & 87,78 \\
\hline Doces & 76,51 & 78,78 \\
\hline \multicolumn{3}{|l|}{ - Reguladores } \\
\hline Frutas & 84,35 & 87,26 \\
\hline Suco de frutas & 92,00 & 89,45 \\
\hline Hortaliças/Folhosos & 52,04 & 56,55 \\
\hline
\end{tabular}

as meninas. A presença dos ovos predomina na alimentação dos adolescentes do sexo masculino quando comparados com o sexo feminino, sendo o ovo frito a forma de consumo freqüentemente empregada. Quando é analisado o consumo de peixe verifica-se estar entre os alimentos de menor freqüência, ocupando posição inferior à lingüiça, alimento que tem alto teor de colesterol. Investigando-se sobre as formas de preparo das carnes predominam as preparações assada ou frita (Tabela 2).

No que se refere ao consumo de alimentos lácteos, as meninas mencionaram ingerir mais leite e queijo quando comparadas aos adolescentes do sexo masculino, exceto para o iogurte, o qual $37,16 \%$ dos meninos declararam consumir. A forma preferida de consumo do leite é em mistura com chocolate em pó, sendo que muitos adolescentes não consomem leite diariamente. Cerca de $25,68 \%$ e $24,57 \%$ de meninos e meninas, respectivamente, não ingerem esse alimento (Tabela 2).

Ainda em relação à Tabela 2, no grupo dos energéticos, predominam os refrigerantes, principalmente entre os adolescentes do sexo masculino, chegando mesmo a superar a posição do arroz. As fontes de gorduras (manteiga, margarina) e preparações gordurosas como batata frita, pizza, lasanha, assim como açúcar, mel, chocolate (tablete), rapadura e sucos industrializados tiveram participação mais importante na dieta dos meninos. Ainda destacam-se os bombons, gelatinas, pudins, cremes, sorvetes, doces e balas, goma de mascar como os itens de maior participação na dieta dos adolescentes do sexo feminino em relação ao sexo masculino. O milho é consumido freqüentemente em preparações como o cuscuz e a mandioca, em preparações como beiju, bolos, biscoito, rosca, mostrando a influência marcante da cultura no hábito alimentar destes jovens. Entretanto, chama a atenção a tendência de substituição de preparações regionais - cuscuz e beiju - que apresentaram menor consumo em relação ao pão branco.

Do grupo dos alimentos reguladores, as frutas tiveram um consumo expressivo (>80\%). Entretanto, verificou-se ser o consumo menos freqüente na forma in natura, com preferência ao consumo de suco de frutas, especialmente entre os meninos, em que 92,00\% dos estudantes mencionaram consumir suco de frutas pelo menos uma vez na semana. As hortaliças folhosos apresentaram um consumo inferior às frutas, demonstrando a necessidade de destacar a importância destes vegetais como componentes alimentares, fontes de fibras, minerais e vitaminas. A freqüência de consumo de hortaliças folhosos entre as meninas $(56,55 \%)$ é maior do que entre os meninos (52,04\%).

Analisando a influência do sexo sobre o consumo semanal de alimentos, pode-se constatar que entre os adolescentes de sexos diferentes houve padrões bastante distintos no consumo de alimentos do grupo dos construtores e energéticos, sugerindo diferentes hábitos e regimes dietéticos (Tabela 3 ). 
Tabela 3. Média e desvio-padrão do consumo semanal* de grupos de alimentos por sexo.

\begin{tabular}{|c|c|c|c|c|c|}
\hline \multirow{2}{*}{ Alimentos } & \multicolumn{2}{|c|}{ Masculino } & \multicolumn{2}{|c|}{ Feminino } & \multirow{2}{*}{$\mathrm{F}^{* *}$} \\
\hline & $x$ & (DP) & $x$ & (DP) & \\
\hline \multicolumn{6}{|l|}{ - Grupo dos Construtores } \\
\hline Carne de frango & $2,82 \pm$ & 1,21 & $2,50 \pm$ & 1,19 & 5,910 \\
\hline Ovos & $2,38 \pm$ & 1,45 & $2,01 \pm$ & 1,44 & 5,345 \\
\hline Leguminosas (ervilha/lentilha) & $1,61 \pm$ & 1,56 & $1,19 \pm$ & 1,41 & 6,489 \\
\hline \multicolumn{6}{|l|}{ - Grupo dos Energéticos } \\
\hline Milho assado/cozido & $1,54 \pm$ & 1,16 & $1,19 \pm$ & 0,97 & 8,963 \\
\hline Bolos/Biscoito/Rosca & $3,33 \pm$ & 1,37 & $3,72 \pm$ & 1,29 & 7,158 \\
\hline Manteiga & $2,76 \pm$ & 1,87 & $2,26 \pm$ & 1,95 & 5,565 \\
\hline Pizza/Lasanha & $2,61 \pm$ & 1,06 & $2,38 \pm$ & 1,02 & 4,305 \\
\hline Gelatinas/pudins/cremes & $1,80 \pm$ & 1,40 & $2,18 \pm$ & 1,46 & 5,972 \\
\hline Sorvete & $2,95 \pm$ & 1,33 & $3,25 \pm$ & 1,34 & 4,313 \\
\hline Sucos industrializados & $1,89 \pm$ & 1,68 & $1,51 \pm$ & 1,64 & 4,510 \\
\hline Refrigerantes & $3,98 \pm$ & 1,15 & $3,55 \pm$ & 1,40 & 9,121 \\
\hline
\end{tabular}

No grupo dos alimentos construtores, os adolescentes do sexo masculino apresentaram consumo alimentar médio significativamente maior $(p<0,05)$ do que as meninas, respectivamente, para a carne de frango: 2,82 e 2,50; ovos: 2,38 e 2,01 e leguminosas: 1,61 e 1,19. A mesma tendência foi observada no grupo dos energéticos, para o milho: 1,54 e 1,19; manteiga: 2,76 e 2,26; pizza/ lasanha: 2,61 e 2,38; sucos industrializados: 1,89 e 1,51 e refrigerantes: 3,98 e 3,55, em que os meninos apresentaram consumo alimentar médio maior em relação às meninas, respectivamente.

Quanto ao consumo alimentar das meninas em relação aos meninos, observou-se média maior ( $p<0,05)$, para bolos, biscoito, rosca: 3,72 e 3,33; gelatinas, pudins, cremes: 2,18 e 1,80 e sorvete: 3,25 e 2,95. Com relação ao restante dos alimentos, não foram encontradas diferenças significativas, o mesmo não ocorrendo entre os grupos dos alimentos reguladores.

Ao se analisar os dados da Tabela 4, observa-se que a idade tem efeito sobre o consumo de alimentos no grupo dos energéticos e no grupo dos reguladores, encontrando-se diferenças significativas importantes entre as diversas faixas etárias. No primeiro grupo, destacam-se o arroz, o milho, os bolos, biscoito, rosca, o mel, o açúcar, a rapadura, as balas, gomas de mascar, o sorvete e o refrigerante, enquanto que no segundo grupo encontram-se as frutas de cores verde-amarelo. Em relação ao arroz, a média de consumo aumentou significativamente com a idade, sendo maior nos adolescentes com 17 a 19 anos. O mesmo não ocorreu com o milho, o mel e a rapadura, cujo consumo diminuiu com a faixa etária dos estudantes.

As informações sobre a merenda oferecida no colégio mostraram associação estatística entre os tipos de lanches e o sexo dos adolescentes. Verifica-se na Figura 1, que os refrigerantes foram mais consumidos pelos adolescentes do sexo masculino enquanto que os salgados/ sanduíches, picolés/sorvetes, além de outros tipos de lanches são mais procurados por estudantes do sexo feminino.

\section{DISCUSSÃO E CONCLUSÃO}

Foi constatado hábito alimentar inadequado para os estudantes adolescentes investigados. Embora haja grande diversificação na alimentação dos escolares estudados, constata-se a presença marcante de alimentos ou preparações gordurosas, alimentos ricos em açúcares com pouca fibra e de menor valor nutricional, como pães, doces, balas, gomas de mascar, bombons, sorvetes, refrigerantes e tabletes de chocolate.

A tendência apresentada pelos adolescentes ao consumo de refeições feitas com gorduras se constitui um motivo de preocupação, tendo em vista que a gordura eleva muito a densidade energética da dieta predispondo, assim, o aparecimento da obesidade e de outras doenças. Isto nos faz supor que as práticas alimentares incorretas observadas neste estudo desempenham um importante papel no ganho de peso apresentado pelos adolescentes. Daí a importância de prestar esclarecimento e orientação aos estudantes no sentido de promover mudanças e melhoria nos seus hábitos alimentares e também na saúde.

A influência do sexo para o risco de sobrepeso não foi identificada neste estudo, diferentemente do observado por Veiga et al. (1996) e Watt \& Sheiham (1996) que demonstraram excesso de peso em meninos, enquanto que Dolinsky et al. (1998), acharam maior prevalência de obesidade nas meninas. 
Tabela 4. Média e desvio-padrão do consumo semanal* de grupos de alimentos por faixa etária.

\begin{tabular}{|c|c|c|c|c|c|c|c|}
\hline \multirow{2}{*}{ Alimentos } & \multicolumn{2}{|c|}{ 10-13 anos } & \multicolumn{2}{|c|}{ 14-16 anos } & \multicolumn{2}{|c|}{ 17-19 anos } & \multirow{2}{*}{$\mathrm{F}^{* *}$} \\
\hline & $x$ & (DP) & $x$ & (DP) & $x$ & (DP) & \\
\hline \multicolumn{8}{|l|}{ - Grupo dos Energéticos } \\
\hline Arroz branco polido & 4,27 & $\pm 1,40$ & $4,48 \pm$ & 1,21 & $4,87 \pm$ & 0,76 & 3,004 \\
\hline Milho assado/cozido & 1,56 & $\pm 1,15$ & $1,08 \pm$ & 0,88 & $0,70 \pm$ & 0,76 & 10,200 \\
\hline Bolos/Biscoito/Rosca & 3,49 & $\pm 1,41$ & $3,77 \pm$ & 1,19 & $3,70 \pm$ & 1,18 & 3,934 \\
\hline Mel & 2,55 & $\pm 1,10$ & $2,52 \pm$ & 0,90 & $2,43 \pm$ & 0,95 & 3,890 \\
\hline Açúcar comum & 1,99 & $\pm 1,58$ & $1,48 \pm$ & 1,23 & $1,91 \pm$ & 2,13 & 5,247 \\
\hline Rapadura & 3,16 & $\pm 1,42$ & $3,04 \pm$ & 1,32 & $3,04 \pm$ & 1,19 & 3,051 \\
\hline Balas/gomas de mascar & 1,34 & $\pm 1,46$ & $0,98 \pm$ & 1,06 & $1,57 \pm$ & 2,43 & 4,537 \\
\hline Sorvete & 3,26 & $\pm 1,49$ & $3,34 \pm$ & 1,42 & $2,96 \pm$ & 1,80 & 6,668 \\
\hline Refrigerantes & 1,84 & $\pm 1,70$ & $1,46 \pm$ & 1,83 & $2,31 \pm$ & 1,72 & 6,134 \\
\hline \multicolumn{8}{|l|}{ - Grupo dos Reguladores } \\
\hline Frutas verde amarelo & 2,91 & $\pm 1,52$ & $3,26 \pm$ & 1,34 & $3,96 \pm$ & 1,19 & 4,144 \\
\hline
\end{tabular}

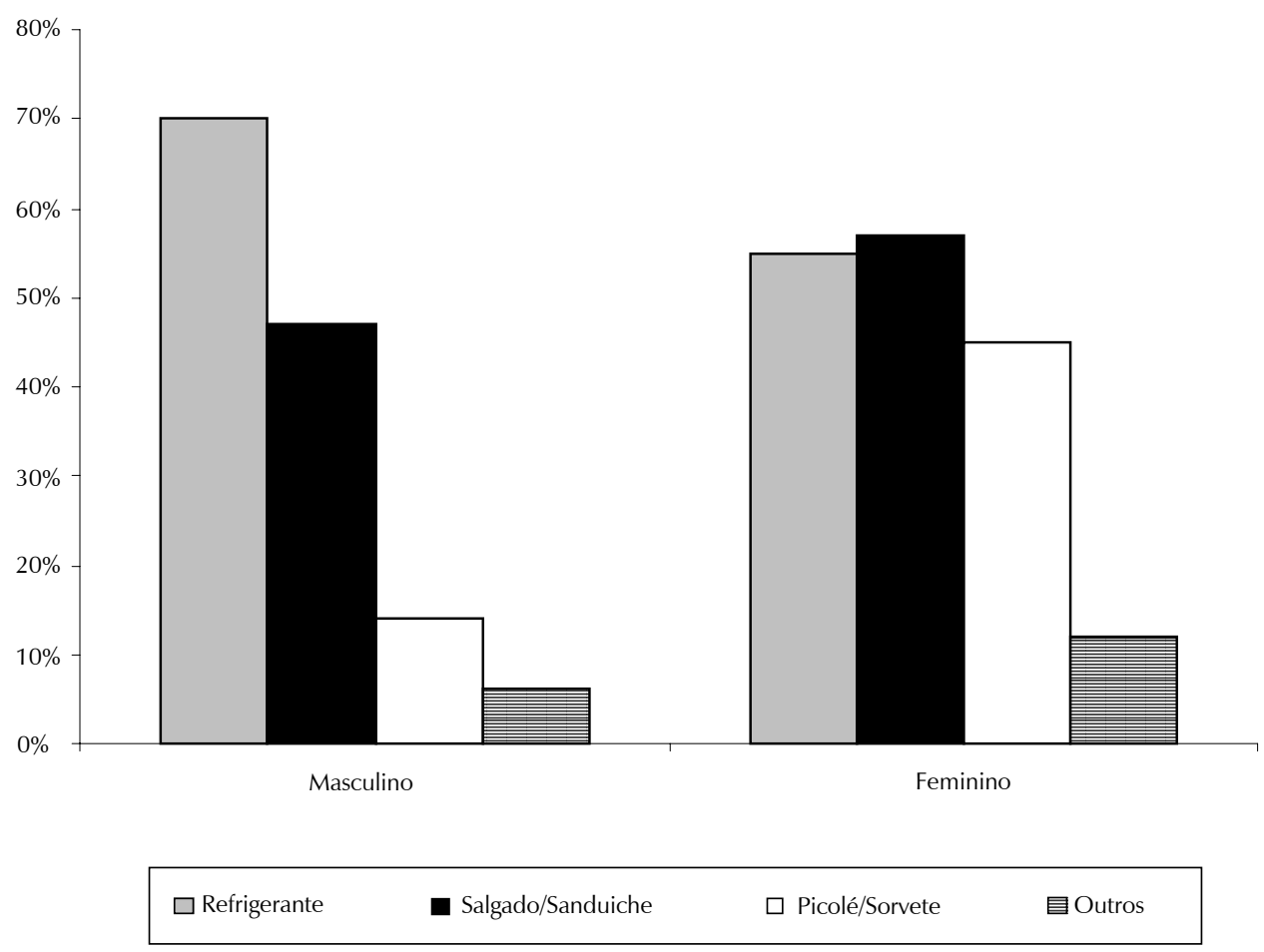

Figura 1. Tipos de merenda realizada no colégio por sexo.

Alguns estudos constatam maior diferença entre indivíduos obesos e não obesos devido precisamente à fonte de energia da alimentação, ou seja, entre os indivíduos obesos as principais fontes de energia são as proteínas e as gorduras, enquanto que os carboidratos contribuem em menor percentual na composição do valor energético total consumido (Borroto et al., 1993; Anta et al., 1996). Este desequilíbrio do perfil energético freqüentemente observado em sociedades desenvolvidas é mais grave entre os indivíduos portadores de obesidade.

Estudos realizados em diferentes países desenvolvidos com adolescentes, mostram que tanto o peso como IMC/Idade elevados se constituem fatores de risco para o desenvolvimento de doenças crônicas (hipertensão arterial, diabetes mellitus), dislipidemias e doenças cardiovasculares e aumentam o risco de morrer 
entre os adultos, devido às complicações características das doenças associadas à obesidade (World Health..., 1995).

Parece não haver dúvidas de que a dieta tem efeito sobre diversas doenças crônicas (Anding et al., 1996). As principais consequências das práticas alimentares incorretas são o aumento no número de diferentes tipos de doenças tais como, obesidade, enfermidades cardiovasculares, hipertensão arterial, diabetes mellitus, osteoporose e anemia (World Health..., 1997), devido ao consumo excessivo de energia, associado ao menor dispêndio de energia ocasionado pelo sedentarismo.

O estudo de Borroto et al. ( 1993) com 80 adolescentes obesos e não obesos, divididos em dois grupos, chama a atenção para a dieta dos obesos, mais rica em gorduras e proteínas com uma maior proporção diária de energia.

Recentemente, uma pesquisa realizada por Anta et al. (1996) sobre hábitos alimentares de adolescentes com sobrepeso mostrou que o maior consumo energético fornecido principalmente pela gordura foi o fator responsável pela obesidade na população pesquisada.

Sabe-se que o padrão alimentar do brasileiro tem sofrido muitas influências e transformações e o estilo da vida moderna tem favorecido o consumo de alimentos industrializados, da alimentação fora de casa e da substituição das refeições tradicionais pelos lanches. Essas mudanças levam ao consumo excessivo de produtos gordurosos, com diminuição no consumo de cereais integrais e aumento no consumo de açúcares, doces e bebidas açucaradas. Os resultados desta pesquisa corroboram com estas informações, sendo este tipo de prática presente na dieta dos adolescentes.

É provável que, para o controle da obesidade e para evitar as enfermidades associadas seja necessário, não só vigiar a ingestão energética, mas também, a composição dos alimentos ingeridos no sentido de se obter alimentação mais saudável. Sabendo-se que na infância se formam os hábitos alimentares, é importante iniciar a educação alimentar neste período de vida para assegurar-se da formação de conduta alimentar satisfatória, evitando o aparecimento da obesidade e suas complicações e melhorar assim a qualidade de vida.

Mudanças no hábito alimentar de uma população é uma tarefa difícil e, na maioria das vezes, requer muito tempo para se obter a conduta alimentar desejada e no escolar, esses comportamentos alimentares inadequados estão associados ao nível socioeconômico e sócio-cultural da família (Cronin et al., 1982; Zacarias et al., 1986), já que a família é responsável pela compra e preparo dos alimentos em casa, transmitindo seus hábitos alimentares às crianças (Gambardella et al., 1999).

A tendência apresentada no consumo de alimentos ricos em açúcares simples, também é motivo de preocupação, tendo em vista os efeitos deletérios destes alimentos quando consumidos de forma desarmoniosa em relação ao conjunto da alimentação. Além de danosos para a saúde oral, os carboidratos simples são fontes de energia vazias mostrando a necessidade de fornecer orientação aos adolescentes sobre o consumo adequado destes alimentos.

O baixo consumo de peixes, ovos e a maior ingestão de frutas em relação às hortaliças corroboram com os resultados dos estudos de Andersen et al. (1995) e Cavadini (1996).

A preferência por merendas não nutritivas encontrada neste estudo também foi constatada por Doyle \& Feldman (1997) em estudantes de classe média de um colégio particular em Manaus, AM, Brasil.

Em função do modismo, da propaganda, influência da escola e dos amigos, da contestação dos valores familiares e sociais, entre tantos outros, os adolescentes facilmente modificam seus hábitos alimentares e, comumente trocam a alimentação habitual pelo consumo de lanches desequilibrados (Farthing, 1991; Gambardella et al., 1999), seguindo o mesmo modelo de consumo elevado de açúcares, proteínas e gorduras de origem animal induzido pela sociedade moderna. Vários trabalhos mostram um baixo consumo de frutas, vegetais, peixes, leite e cereais, enquanto para outros alimentos ricos em proteínas, gorduras e açúcares ocorre o inverso (McPherson et al., 1990; Strain et al., 1994; Pérez-Liamas et al., 1996).

Muitas vezes os adolescentes consomem lanches e fast foods ricos em energia, contribuindo para o ganho de peso; outras vezes, tendem a pular refeições, comportamento mais freqüentemente observado entre as meninas com o objetivo de perder peso (Sargent et al., 1994; Andersen et al., 1995; French et al., 1995).

É bom lembrar que o grupo dos laticínios encontra-se entre os alimentos com baixa freqüência de consumo entre os adolescentes. O leite e seus derivados são excelentes fontes de cálcio, dentre outros nutrientes, daí recomendar-se o consumo diário de, pelo menos, três porções de leite, queijo ou iogurte para atender às necessidades do mineral na adolescência. É na adolescência que ocorre o estirão do crescimento linear com simultâneo aumento da massa óssea que tem no cálcio, elemento fundamental para que o processo ocorra a contento.

Dados de várias pesquisas mostram associação positiva entre a quantidade de cálcio da dieta na fase de crescimento e puberdade, como fator de prevenção da perda da massa óssea em mulheres na pós-menopausa (Mortensen \& Charles, 1996; Nieves et al., 1998).

Tendo em vista a relação entre consumo de cálcio na puberdade e adolescência e o risco de osteoporose, o baixo consumo de leite e derivados é uma preocupação em relação aos adolescentes desta pesquisa, principalmente entre as meninas que têm maior probabilidade de desenvolver a doença na vida adulta. É bom lembrar que, apesar do leite estar com baixa freqüência de consumo pelos adolescentes, observa-se a tendência de aumento no consumo do alimento pelas meninas. 
Algumas investigações têm descrito que os hábitos alimentares dos adolescentes são inadequados, com baixa freqüência de produtos lácteos, verduras e frutas (Boneu et al., 1994) e excesso de gordura e açúcar (Gambardella, 1996). Outras pesquisas têm demonstrado que os alimentos mais consumidos pelos adolescentes são leite, carne, aves e pão (Schorr et al., 1972; Stewart \& Darnton-Hill, 1981).

Apesar da limitação apresentada pelo método no que se refere a quantificação dos nutrientes ingeridos, as questões aqui apresentadas e analisadas sobre a freqüência de consumo de grupos de alimentos mostram algumas práticas inadequadas dos adolescentes. Alimentos importantes e de consumo diário aparecem com baixa freqüência semanal, como leite e derivados, hortaliças e vegetais folhosos, principalmente. As frutas são mais consumidas na forma de suco e houve pouca preferência pelo peixe. Tendo em vista a importância nutricional de vitaminas, minerais, fibras, ácidos graxos w-3 e substâncias antioxidantes presentes em muitos destes alimentos, pode-se inferir que os adolescentes estudados podem vir a apresentar alterações nutricionais devido às deficiências alimentares nestes constituintes. Meninos e meninas apresentaram padrões de consumo diferenciados, havendo maior freqüência de consumo, entre os meninos, para os alimentos energéticos (milho, manteiga, pizza, lasanha, sucos industrializados, refrigerantes) e construtores (frango, ovos, leguminosas tipo ervilha, lentilha). Assim, levando em consideração os resultados encontrados, bem como as observações feitas no que se refere aos alimentos dos três grupos (construtores, energéticos reguladores), julga-se necessário dedicar mais atenção aos cuidados nutricionais também para este segmento da população.

Em função do risco de sobrepeso existir no grupo de adolescentes pesquisados, reforça-se a necessidade de estratégias de prevenção, no sentido de promover mudanças de hábitos e comportamentos voltados, não só para a dieta, mas principalmente, para o estilo de vida, antes da evolução dos problemas e agravos à saúde.

Para melhor conhecer as diferenças nas práticas alimentares entre os estudantes, faz-se necessário estudos posteriores para que, no desenvolvimento das estratégias de educação nutricional, se formulem propostas mais efetivas.

\section{AGRADECIMENTOS}

Os autores agradecem à direção do colégio, pelas facilidades outorgadas para a realização do estudo; aos adolescentes pela sua participação.

\section{REFERÊNCIAS BIBLIOGRÁFICAS}

ANDERSEN, L.F., NES, M., SANDSTAD, B., BJORNEBOE, G.E., DREVON, C.A. Dietary intake among Norwegian adolescents. European Journal of Clinical Nutrition, London, v.49, n.8, p.55-64, 1995.
ANDING, J.D., KUBENA, K.S., McINTOSH, W.A., O'BRIEN, B. Blood lipids, cardiovascular fitness, obesity, and blood pressure: the presence of potencial coronary heart disease risk factors in adolescents. Journal American of the Dietetic Association, Chicago, v.96, n.3, p.238-242, 1996.

ANTA, R.M.O., CARVAJALES, P.A., MARCOS, A.M.R., SOBALER, A.M.L., SOBRADO, R.R.R., GONZÁLEZ-FERNÁNDEZ, M. Hábitos alimentares y ingesta de energia y nutrientes en adolescentes com sobrepeso en comparación com los de peso normal. Análes Españoles de Pediatria, Madrid, v.44, n.3, p.203-8, 1996.

BONEU, M., GÓMEZ-QUIRANTE, A., FEIJÓO, N., MARTOS, C., FOZ, G. Hábitos alimentarios y frecuencia de consumo de alimentos en los escolares de octavo de EGB de un Área Básica de Salud de Mataró. Atención Primaria, Barcelona, v.14, n.2, p.591-5, 1994.

BORROTO, J.M., RAMOS, L.T., MOROÑO, M., HERMELO, C.M.M., BACALLAO, J., AMADOR, C.M.M. Ingreso energético en ñino y adolescentes obesos. Revista Cubana Pediatric, Havana, v.65, n.3, p.165-75, 1993.

CAVADINI, C. Dietary habits adolescence: contribution of snacking. In: FEEDING from toddlers to adolescence. Philadelphia : Lippincott-Raven Publishers, 1996. (Nestlé Nutrition Workshop Series, 37).

CRONIN, F.J., KREBS-SMITH, S.M., WYSE, B.W., LIGTH, L. Characterizing food usage by demographic variables. Journal of the American Dietetic Association, Chicago, v.81, n.6, p.661-673, 1982.

DOLINSKY, M., GUIMARÃES, J.P., SILVA, C.C.C.V., COSTA, C.R.C., CINTRA, P.P. Estado Nutricional de crianças e adolescentes do município de Altamira/Maranhão. In: CONGRESSO BRASILEIRO DE NUTRIÇÃO, 15., 1998, Brasília. Anais, Brasília : Associação Brasileira de Nutrição, 1998. p.186.

DOYLE, E.I., FELDMAN, R.H.L. Factors affecting nutrition behavior among middle-class adolescents in urban area of Northern region of Brazil. Revista de Saúde Pública, São Paulo, v.3, n.1, p.342-350, 1997.

FARTHING, M.C. Current eating patterns of adolescents in the United States. Nutrition Today, Baltimore, v.26, n.2, p.35-39, 1991.

FRENCH, S.A., STORY, M., DOWNES, B., RESNICK, M.D., BLUM, R.W. Frequent dieting among adolescents: psychosocial and health behavior correlates. American Journal of Public Health, Washington DC, v.85, n.5, p.695-701, 1995.

GAMBARDELLA, A.M.D. Adolescentes estudantes de período noturno: como se alimentam e gastam suas energias. São Paulo, 1996. p.42-60. Tese (Doutorado) - Faculdade de Saúde Pública, Universidade de São Paulo, 1996.

GAMBARDELLA, A.M.D., FRUTUOSO, M.F.P., FRANCHI, C. Prática alimentar de adolescentes. Reviews Nutrition, New York, v.12, n.1, p.55-63, 1999.

GUO, S.S., ROCHE, A.F., CHUMLEA, W.C., GARDNER, J.D., SIERVOGEL, R.M. The predictive value of chilhood body mass index values for overweight at age 35 years. American Journal of Clinical Nutrition, Bethesda, v.59, n.4, p.810-819, 1994.

HUANG, YA-LI., SONG, W.O., SCHEMMEL, R.A., HOERR, S.M. What do college students eat? Food selection and meal pattern. Nutrition Research, Tarrytown NY, v.14, n.8, p.1143-1153, 1994. 
LERNER, B.R. Alimentação e a anemia carencial em adolescentes. São Paulo, 1994. p.26-77. Tese (Doutorado) - Faculdade de Saúde Pública, Universidade de São Paulo, 1994.

MCPHERSON, R.S., NICHAMAN, M.Z., KOLH, H.W., REED, D.B., LABARTHE, D.R. Intake and food sources of dietary fat among schoolchildren in The Woodlands. Pediatric, Evanston, v.86, n.4, p.520-526, 1990.

MONTEIRO, C.A., MONDINI, D. Mudanças no padrão de alimentação. In: MONTEIRO, C.A. Velhos e novos males de saúde no Brasil. São Paulo : Hucitec, 1995. p.79-89.

MORTENSEN, L., CHARLES, P. Bioavailability of calcium supplements and the effect of vitamin D: comparisons between milk, calcium carbonate, and calcium carbonate plus vitamin D. American Journal of Clinical Nutrition, Bethesda, v.63, n.3, p.354-357, 1996.

MUST, A., JACQUES, P.F., DALLAL, G.E., BAJEMA, C.Y., DIETZ, W.H. Long-term morbidity and mortality of overweight adolescents. A follow-up at the Harvard Growth Study of 1922 to 1935. New England Journal of Medicine, Boston, v. 327, n.19, p. 1350-1355, 1992.

NIEVES, J.W., KOMAR, L., COSMAN, F., LIBDSAY, R. Calcium potentiates the effect of estrogen and calcitonin on bone mass: review and analysis. American Journal of Clinical Nutrition, Bethesda, v.67, n.1, p.18-24, 1998.

PÉREZ-LIAMAS, F., GARAULET, M., NIETO, M., BARAZA, J.C., ZAMORA, S. Estimates of food intake and dietary habits in a radom sample of adolescents in south-east Spain. Journal of Human Nutrition and Dietetics, Oxford, v.9, n.6, p.451-461, 1996.

SARGENT, R.G., KEMPER, K.A., SCHULKEN, E. Dietary behaviors of South Carolina adolescents. Journal South Carolina Medical Association, Greenville SC, v.90, n.6, p.263-269, 1994.
SCHORR, B., SANJUR, D., ERICKSON, E. Teen-age food habits. Journal of the American Dietetic Association, Chicago, v.61, n.4, p.415-420, 1972.

STEWART, A.T., DARNTON-HILL. Food habits of adolescents. Nutrition Reviews, New York, v.39, n. 2, p.73-88, 1981.

STRAIN, J.J., ROBSON, P.J., LIVINGSTONE, M.B.E., PRIMROSE, E.D., SAVAGE, J.M., CRAN, G.W., BOREHAM, C.A.G. Estimates of food and macronutrient intake in a random sample of Northern Island adolescents. British Journal of Nutrition, London, v.72, n.3, p.43-52, 1994.

VEIGA, G.V., SANTOS, P.P., COUTINHO, D.S., CORÉ, M.F.R., PAES, E.C. Avaliação de obesidade em adolescentes através do Índice de Massa Corporal. In: CONGRESSO NACIONAL DA SOCIEDADE BRASILEIRA DE ALIMENTAÇÃO E NUTRIÇÃO, 4., 1996. Brasília. Livro de Resumos. Brasília : Sociedade Brasileira de Alimentação e Nutrição, 1996, SP-36.

WATT, R.G., SHEIHAM, A. A Dietary patterns and changes in inner city adolescents. Journal of Human Nutrition and Dietetics, Oxford, v.9, n.6, p.451-461, 1996.

WORLD HEALTH ORGANIZATION, Obesity: preventing and managing the global epidemic. Geneva, 1997. p.276. (Report of WHO Consultation on Obesity; 3-5 june).

WORLD HEALTH ORGANIZATION. Physical status: the use and interpretation of anthropometry, Genebra, 1995. p.263-311.

ZACARIAS, I., AGAYO, M., VÁSQUEZ, M., BALLESTER, D., IVANOVIC, D. Hábitos alimentarios de estudiantes que egresan de educación media en el area metropolitana de Santiago. Chile. Archivos Latinoamericanos de Nutrición, Guatemala, v.36, n.2, p.268-281, 1986.

Recebido para publicação em 14 de julho de 1999 e aceito em 10 de outubro de 2000. 